\title{
Imperfect Barotropic Magnetic Tilted Cosmological Model
}

\author{
Anita Bagora and Rakeshwar Purohit
}

\begin{abstract}
Tilted Bianchi type I cosmological model in the presence of magnetic field and barotropic fluid is investigated. To determine complete solution, we have assumed that the condition $p=\gamma \in$, where $\mathbf{p}$ being isotropic pressure, $\in$ the matter density .It has been assumed that the expansion in the model is only in two directions i.e. one of the component of Hubble parameter is zero. Also, we have seen that Maxwell's equations $F[i j ; k]=0$ is satisfied by $F 23=$ constant. The physical and geometrical aspects of the model in the presence and absence of magnetic field together with singularity in the model are also discussed.
\end{abstract}

Index Terms-Tilted cosmological model, magnetic field; bianchi type-I universe. PACS numbers: 98.80.-k, 04.20.-q 2000 Mathematics Subject Classification: 83C50, $83 F 05$.

\section{INTRODUCTION}

Bianchi type I cosmological models which are the anisotropic generalization of FRW models with flat space slices have been widely studied. The evidence for an intergalactic homogeneous magnetic field speculated to be of primordial nature, has prompted a number of workers to successfully incorporate a homogeneous magnetic field in Bianchi type I spaces. Throne [1] investigated LRS Bianchi type I cosmological model containing a magnetic field directed along one axis with a barotropic fluid. Magnetic Bianchi type I cosmological models satisfying a barotropic equation of state have considered by Jacobs [2-3]. A qualitative analysis of Bianchi type I models with a magnetic field has also been given by Collins [4]. Hughston and Jacobs [5] have shown that the existence of a homogeneous primordial magnetic field in the universe. Desikan [6] has obtained some models of a FRW universe filled with imperfect fluid having bulk viscosity in the framework of Brans-Dicke Theory, using the full Israel-Stewart-Hiscock causal theory. The non orthogonal Bianchi universe gives rise to tilted Bianchi cosmology. A lot of work has been done for tilted models, in such models fluid flow are non-orthogonal to the hyper surfaces of homogeneity. Investigators have shown a considerable interest in spatially homogeneous and anisotropic universe in which the fluid is tilted. The general dynamics to tilted models have been studied by King and Ellis [7], Ellis and King [8], Collins and Ellis [9], Coley [10]. Dunn and Tupper [11] investigated that

Manuscript received May 18, 2001; revised June 13, 2012

A. Bagora is with the Jaipur National University, Jaipur-302025, India (e-mail: anitabagora@gmail.com).

R. Purohit is with the M.L. Sukhadia University, Udaipur-313001, India (e-mail: ramkrishnadr@gmail.com). tilting universe are possible when electromagnetic field is present.Bianchi type I cosmological models have been extensively studied in the past especially as example of the homogeneous, shearing tilted universe by Mukherjee [12]. Exact tilted cosmological solutions satisfying the combined perfect fluid electromagnetic field equation have been given by Lorenz [13-14]. Bagora et al. [15] have also investigated magnetized Bianchi type I homogeneous cosmological models with disordered radiation. Bagora [16-17] obtained tilted homogeneous cosmological model in different context. Cosmological models with heat flows have been studied by a number of auhors, viz., Novello and Reboucas[18], Ray[19],Coley and Tupper[20] and Deng[21]. Motivated by these studies, in this paper, we propose to investigate some tilted homogeneous cosmological model for barotropic fluid in the presence and absence of magnetic field. It has been shown that tilted nature of the model is preserved due to magnetic field. The magnetic field is due to an electric current produced along $\mathrm{X}$-axis. Also, it has been assumed that the expansion in the model is only in two directions i.e. one of the component of Hubble parameter is zero. The various physical and geometrical aspects of the model are discussed.

\section{Metric And Field EQuations}

We consider the Bianchi type-I metric in the form

$$
\mathrm{ds}^{2}=-\mathrm{dt}^{2}+\mathrm{dx}^{2}+B^{2} \mathrm{dy}^{2}+C^{2} \mathrm{dz}^{2}
$$

where $B$ and $C$ are functions of ' $t$ ' alone.

The energy-momentum tensor for perfect fluid distribution with heat conduction is taken into the form by Ellis [22] as

$$
T_{i}^{j}=(\in+p) v_{i} v^{j}+p g_{i}^{j}+q_{i} v^{j}+v_{i} q^{j}+E_{i}^{j}
$$

together with

$$
\begin{gathered}
g_{i j} v_{i} v^{j} \tilde{=} 1, \\
q_{i} q^{j}>0, \\
q_{i} v^{i}=0 .
\end{gathered}
$$

Here $\mathrm{E}_{\mathrm{i}}^{\mathrm{j}}$ is the electromagnetic field given by Lichnerowicz [23] as

$$
E_{i}^{j}=\bar{\mu}\left[|h|^{2}\left(v_{i} v^{j}+\frac{1}{2} g_{i}^{j}\right)-h_{i} h^{j}\right]
$$

where $\bar{\mu}$ is magnetic permeability and $h_{i}$ is the magnetic flow vector defined by 


$$
h_{i}=\frac{\sqrt{-g}}{2 \bar{\mu}} \in_{i j k \ell} F^{k \ell} v^{j}
$$

Here $\mathrm{F}_{\mathrm{k} \ell}$ is the electromagnetic field tensor and $\epsilon_{\mathrm{ijk} \ell}$ the Levi-Civita tensor density.

From (7) we find that $h_{1} \neq 0, h_{2}=0, h_{3}=0, h_{4} \neq 0$. This leads to $F_{12}=0=F_{13}$ by virtue of (7). We also find that $F_{14}=0=$ $F_{24}$ due to the assumption of infinite conductivity (Maartens[24])of the fluid. We take the incident magnetic field to be in the direction of $\mathrm{x}$-axis so that the only non-vanishing component of $F_{i j}$ is $F_{23}$.

The first set of Maxwell's equation

$$
F_{i j ; k}+F_{j k ; i}+F_{k i ; j}=0
$$

leads to

$F_{23}=$ constant $H$ (say)

Now $h_{1}=\frac{H}{\bar{\mu} B C} \cosh \lambda, h_{4}=\frac{H}{\bar{\mu} B C} \sinh \lambda$

Since

$$
\begin{gathered}
|h|^{2}=h_{\ell} h^{\ell}=h_{1} h^{1}+h_{4} h^{4}=g^{11}\left(h_{1}\right)^{2}+g^{44}\left(h_{4}\right)^{2} \\
=\frac{H^{2} \cosh ^{2} \lambda}{\bar{\mu}^{2} B^{2} C^{2}}-\frac{H^{2} \sinh ^{2} \lambda}{\bar{\mu}^{2} B^{2} C^{2}}=\frac{H^{2}}{\bar{\mu}^{2} B^{2} C^{2}}
\end{gathered}
$$

Equation (6) leads to

$$
E_{1}^{1}=\frac{-H^{2}}{2 \bar{\mu} B^{2} C^{2}}=-E_{2}^{2}=-E_{3}^{3}=E_{4}^{4}
$$

Here $p$ is the pressure, $\in$ the density, $q^{i}$ the heat conduction vector orthogonal to $v^{i}$. The fluid flow vector $v^{i}$ has the components $(\sinh \lambda, 0,0, \cosh \lambda)$ satisfying (3) and $\lambda$ is the tilt angle.

The Einstein's field equation

$$
R_{i}^{j}-\frac{1}{2} R g_{i}^{j}=-8 \pi T_{i}^{j} . \quad(c=G=1)
$$

The field equation for the line element (1) leads to

$$
\begin{aligned}
\frac{B_{44}}{B}+\frac{C_{44}}{C}+\frac{\mathrm{B}_{4} C_{4}}{\mathrm{BC}} & =-8 \pi\left[\begin{array}{l}
(\in+p) \sinh ^{2} \lambda+p+2 q_{1} \sinh \lambda- \\
\frac{H^{2}}{2 \bar{\mu}(B C)^{2}}
\end{array}\right] \\
\frac{C_{44}}{C} & =-8 \pi\left[p+\frac{H^{2}}{2 \bar{\mu}(B C)^{2}}\right] \\
\frac{B_{44}}{B} & =-8 \pi\left[p+\frac{H^{2}}{2 \bar{\mu}(B C)^{2}}\right]
\end{aligned}
$$

$$
\frac{B_{4} C_{4}}{B C}=-8 \pi\left[-(\in+p) \cosh ^{2} \lambda+p-2 q_{1} \sinh \lambda-\frac{H^{2}}{2 \bar{\mu}(B C)^{2}}\right]
$$

$$
(\in+p) \sinh \lambda \cosh \lambda+q_{1} \cosh \lambda+q_{1} \frac{\sinh ^{2} \lambda}{\cosh \lambda}=0
$$

where the suffix ' 4 ' stands for ordinary differentiation with respect to the cosmic time ' $t$ ' alone.

\section{Solution Of The FiEld EQuATIONS}

Equations from (9)-(13) are five equations in six unknown $B, C \in, p, \mathrm{q}_{1}$ and $\lambda$.For the complete determination of these quantities, we assume that the model is filled with barotropic perfect fluid which leads to

$$
p=\gamma \in
$$

where $0 \leq \gamma \leq 1$.

Equations (9) and (12) lead to

$$
\frac{B_{44}}{B}+\frac{C_{44}}{C}+\frac{2 \mathrm{~B}_{4} \mathrm{C}_{4}}{\mathrm{BC}}=8 \pi(\in-p)+\frac{8 \pi H^{2}}{\bar{\mu}(B C)^{2}}
$$

By using (14) in (15), we have

$$
\frac{B_{44}}{B}+\frac{C_{44}}{C}+\frac{2 \mathrm{~B}_{4} \mathrm{C}_{4}}{\mathrm{BC}}=-8 \pi\left(p-\frac{p}{\gamma}\right)+\frac{8 \pi H^{2}}{\bar{\mu}(B C)^{2}}
$$

Using (11) in equation (13), we have

$$
\begin{aligned}
& \frac{B_{44}}{B}+\frac{C_{44}}{C}+\frac{2 \mathrm{~B}_{4} \mathrm{C}_{4}}{\mathrm{BC}}=\left[\frac{B_{44}}{B}+\frac{8 \pi H^{2}}{2 \bar{\mu}(B C)^{2}}\right]\left(1-\frac{1}{\gamma}\right)+ \\
& \frac{8 \pi H^{2}}{\bar{\mu}(B C)^{2}}
\end{aligned}
$$

Equations (7) and (8) lead to

$$
\frac{B_{44}}{B}-\frac{C_{44}}{C}=0
$$

Let us assume that $B C=\mu, \frac{\mathrm{B}}{C}=\nu$

Using (19) in equation (18),we have

$$
\frac{\nu_{4}}{\nu}=\frac{a}{\mu}
$$

where ' $a$ ' is constant of integration.

Using (19) and (20) in equation (17), we have

$$
\mu_{44}+\frac{s \mu_{4}^{2}}{\mu}=\frac{s a^{2}}{\mu}+\frac{\ell K}{\mu}
$$


Here $\quad s=\frac{1}{4}\left(\frac{\gamma-1}{\gamma+1}\right), \quad \ell=\frac{3 \gamma+1}{\gamma+1}, \quad K=\frac{4 \pi H^{2}}{\bar{\mu}}$

Equation (21) gives

$$
K=\frac{4 \pi H^{2}}{\bar{\mu}}
$$

where ' $b$ ' is constant of integration and $\mu_{4}=f(\mu)$.

Equation (20) leads to

$$
\log \nu=\int \frac{\mathrm{a} \sqrt{\mathrm{s}} \mu^{\mathrm{s}} \mathrm{d} \mu}{\mu \sqrt{a^{2} s \mu^{2 \mathrm{~s}}+\ell K \mu^{2 \mathrm{~s}}+s b}}
$$

Hence the metric (1) reduces to the form

$$
\mathrm{ds}^{2}=-\frac{\mathrm{d} \mu^{2}}{\mathrm{f}^{2}}+d x^{2}+\mu \nu d y^{2}+\frac{\mu}{\nu} d z^{2}
$$

The metric (24) reduces to the form

$$
\mathrm{ds}^{2}=\frac{-\mathrm{s} T^{2 \mathrm{~s}} \mathrm{~d} T^{2}}{a^{2} s T^{2 \mathrm{~s}}+\ell K T^{2 \mathrm{~s}}+s b}+d X^{2}+\mathrm{T} \nu \mathrm{dY^{2 }}+\frac{\mathrm{T}}{\nu} d Z^{2}
$$

where $v$ is determined by (23) when $\mu=T$ and $x=X, y=Y$ $z=Z$.

In the absence of magnetic field, the metric (25) becomes

$$
\mathrm{ds}^{2}=\frac{-T^{2 \mathrm{~s}} \mathrm{~d} T^{2}}{a^{2} T^{2 \mathrm{~s}}+b}+d X^{2}+\mathrm{T} \nu \mathrm{dY} \mathrm{Y}^{2}+\frac{\mathrm{T}}{\nu} d Z^{2}
$$

where $\nu$ is determined by (23) when $\mu=T$ and $K=0$.

\section{Some Physical and Geometrical Properties}

The matter density $\in$ and isotropic pressure $\mathrm{p}$ for the model (25) are given by

$$
\begin{aligned}
& 8 \pi p=\frac{\left[16 \gamma(\gamma+1) K T^{2 s}+(3 \gamma+1)(\gamma-1)\right]}{8(\gamma+1)(\gamma-1) T^{2 s+2}} \\
& 8 \pi \in=\frac{\left[16 \gamma(\gamma+1) K T^{2 s}+(3 \gamma+1)(\gamma-1)\right]}{8 \gamma(\gamma+1)(\gamma-1) T^{2 s+2}}
\end{aligned}
$$

The tilt angle $\lambda$ is given by

$$
\begin{array}{r}
\cosh \lambda=\frac{1}{2} \sqrt{\frac{\psi_{1}}{\psi_{3}}} \quad \text { (29) } \quad \psi_{1}=2(11 \gamma+7)(\gamma+1) K T^{2 s}+(3 \gamma+1)(\gamma-1)(\gamma+3), \\
\psi_{2}=2(5 \gamma+9)(\gamma+1) K T^{2 s}+(3 \gamma+1)(\gamma-1)^{2}, \\
\psi_{3}=(3 \gamma-1)(\gamma+1) K T^{2 s}+(3 \gamma+1)(\gamma-1),
\end{array}
$$

The scalar of expansion $\theta$ calculated for the flow vector $v^{i}$ for the model (25) is given by

$$
\theta=\frac{\psi_{4}}{8 \mathrm{~T}^{\mathrm{s}+1}} \sqrt{\frac{(\gamma-1) a^{2} T^{2 s}+4(3 \gamma-1) K T^{2 s}+(\gamma-1) \mathrm{b}}{(\gamma-1) \psi_{1} \psi_{3}^{3}}}
$$

The flow vectors $v^{i}$ and heat conduction vectors $q_{\mathrm{i}}$ for the model (24) are given by

$$
\begin{aligned}
& \nu^{1}=\frac{1}{2} \sqrt{\frac{\psi_{2}}{\psi_{3}}} \\
& v^{4}=\frac{1}{2} \sqrt{\frac{\psi_{1}}{\psi_{3}}}
\end{aligned}
$$

$$
q_{1}=\frac{-\psi_{1}}{256(\gamma+1)(\gamma-1) \pi \gamma \mathrm{T}^{2 \mathrm{~s}+2}} \sqrt{\frac{\psi_{2}}{\psi_{3}}}
$$

$$
q_{4}=\frac{\psi_{1}}{256(\gamma+1)(\gamma-1) \pi \gamma \mathrm{T}^{2 \mathrm{~s}+2}} \sqrt{\frac{\psi_{1}}{\psi_{3}}}
$$

The non-vanishing components of shear tensor $\left(\sigma_{\mathrm{ij}}\right)$ and rotation tensor $\left(\sigma_{\mathrm{ij}}\right)$ are given by

$\sigma_{11}=\frac{\psi_{5}}{48 \mathrm{~T}^{\mathrm{s}+1}} \sqrt{\frac{\left[(\gamma-1) a^{2} T^{2 s}+4(3 \gamma-1) K T^{2 s}+(\gamma-1) \mathrm{b}\right] \psi_{1}}{(\gamma-1) \psi_{3}^{5}}}$

$\sigma_{14}=\frac{-\psi_{5}}{48 \mathrm{~T}^{\mathrm{s}+1}} \sqrt{\frac{\left[(\gamma-1) a^{2} T^{2 s}+4(3 \gamma-1) K T^{2 s}+(\gamma-1) \mathrm{b}\right] \psi_{2}}{(\gamma-1) \psi_{3}{ }^{5}}}$

$\omega_{14}=\frac{-(3 \gamma-1)(\gamma-1)\left(3 \gamma^{2}-14 \gamma-17\right)}{8 \mathrm{~T}^{s-1}} \sqrt{\frac{\left[(\gamma-1) a^{2} T^{2 s}+4(3 \gamma-1) K T^{2 s}+(\gamma-1) \mathrm{b}\right]}{(\gamma-1) \psi_{3}^{3} \psi_{2}}}$.

Thus $\quad \sigma_{11} \nu^{1}+\sigma_{14} \nu^{4}=0$

Here 


$$
\begin{gathered}
\left(9 \gamma^{3}+149 \gamma^{2}+127 \gamma+3\right) K T^{2 s}, \\
\psi_{5}=4(11 \gamma+7)(3 \gamma-1)(\gamma+1)^{2} K^{2} T^{4 s}+2(3 \gamma+1)^{2}(\gamma-1)^{2}(\gamma+3)+(3 \gamma+1)(\gamma-1)
\end{gathered}
$$

$$
\left(3 \gamma^{3}+83 \gamma^{2}+64 \gamma-16\right) K T^{2 s}
$$

The rates of expansion $H_{i}$ in the directions of $y$ and $z$ - axes are given by

$$
\begin{gathered}
H_{2}=\frac{1}{2 T}\left[\frac{\sqrt{a^{2} s T^{2 \mathrm{~s}}+\ell K T^{2 \mathrm{~s}}+s b}}{T^{s}}+a\right] \\
\left.H_{3}=\frac{1}{2 T}\left[\frac{\sqrt{a^{2} s T^{2 \mathrm{~s}}+\ell K T^{2 \mathrm{~s}}+s b}}{T^{s}}-a\right]\right]
\end{gathered}
$$

\section{CONCLUSION}

The model (25) represents a tilted model, $\in \rightarrow \infty$ when $T \rightarrow 0$ and $\gamma<0$.The model starts with a big-bang at $T=0$ and the expansion in the model decreases as time increases. Also, $\sigma_{i j} \nu^{j}=0$ is satisfied as $\sigma_{11} \nu^{1}+\sigma_{14} \nu^{4}=0$. The model has cigar type singularity at $T=0$ when $s>0$ and it has point type singularity at $T=0$ when $s<0$ (MacCallum, [25]).

In the absence of magnetic field, for $s<0, \in \rightarrow \infty$ when $T \rightarrow 0$ and $\in \rightarrow 0$ when $T \rightarrow \infty$, therefore $\in$ is the decreasing function of time. However, in the absence of magnetic field the tilt angle $\lambda=0$.Thus magnetic field preserve the tilted nature of the model. $\infty$. Since $\begin{aligned} & L t \\ & T \rightarrow \infty\end{aligned}$ $\left(\frac{\sigma}{\theta}\right) \neq 0$, the model does not approach isotropy for large values of $T$. The x-component of Hubble parameter is zero due to the assumption of metric. However density $\in=0, x, y$ and $z$ components to Hubble parameters are zero at $T=\infty$, thus the metric as asymptotically empty.

\section{REFERENCES}

[1] K. S. Throne. Astrophys. J. vol. 148, no. 51, 1967.

[2] K. C. Jacobs. Astrophys. J. vol. 153, no. 661, 1968.

[3] K. C. Jacobs. Astrophys. J., vol. 155, no. 379, 1969.

[4] C. B. Collins. Comm. Math. Phys. vol. 27, no. 37, 1972.

[5] L. D. Hughston and K. C. Jacobs. Astrophys. J. vol. 160, no. 147, 1970

[6] K. Desikan, Pramana J. Phys. vol. 45, no. 6, pp. 511, 1995.

[7] A. R. King and G. F. R. Ellis. Comm. Math. Phys. vol. 31, no. 209, 1973.

[8] G. F. R. Ellis and A. R. King. Comm. Math. Phys. vol. 38, no. 119. 1974.

[9] C. B. Collins and G. F. R. Ellis. Phys. Rev. vol. 56, no. 65, 1979.

[10] A. A. Coley. Phys. Lett. A. vol. 137, no. 235, 1989.

[11] K. A. Dunn and B. O. J. Tupper. Astrophy. J. vol. 222, no. 405, 1978.

[12] G. Mukherjee. J. Astrophys. Astron. vol. 4, no. 295, 1983.

[13] D. Lorenz. Phys. Lett. vol. 83A, no. 155, 1981.

[14] D. Lorenz. Gen. Rela. Gravit., vol. 13, no. 795, 1981.

[15] A. Bagora. EJTP. vol. 14, pp. 373-382, 2010.

[16] A. Bagora, G. S. Rathore, and S. Gandhi. Rom. J. Phys. vol. 54, no. 7-8, pp. 777-786, 2009.

[17] A. Bagora. Astrophys. Space Sci. vol. 319, no. 155, 2008.

[18] M. Novello and M. J. Reboucas. Astroph. J. vol. 225, no. 719, 1978.

[19] D. Ray. J.Math. Phys. vol. 21, no. 2797, 1980.

[20] A. A. Coley and B. O. J. Tupper. Astrophys. J. vol. 280, no. 26, 1984.

[21] Y. Deng. Gen.Rela.Gravit. vol. 21, no. 503, 1989.

[22] G. F. R. Ellis, "General Relativity and cosmology,” R. K. Sachs (Ed), Academy Press, New York, 1971, pp. 116.

[23] A. Lichnerowicz, "Relativistic Hydrodynamics," Benjamin, New York, 1967, pp. 13.

[24] R. Maartens. Pramana J. Phys. vol. 55, no. 576, 2000.

[25] M. A. H. Mac Callum. Comm. Maths. Phys. vol. 20, no. 57, 1971. 